\title{
THE INITIAL-VALUE PROBLEM FOR THE KELVIN-HELMHOLTZ INSTABILITIES OF HIGH-VELOCITY AND MAGNETIZED SHEAR LAYERS
}

\author{
$\mathrm{BY}$ \\ S. ROY CHOUDHURY \\ Department of Mathematics, University of Central Florida, Orlando, FL
}

\begin{abstract}
The general initial-value problem for the linear Kelvin-Helmholtz instability of arbitrarily compressible velocity shear layers is considered for both the unmagnetized and magnetized cases. The time evolution of the physical quantities characterizing the layer is treated using Laplace transform techniques. Singularity analysis of the resulting equations using Fuchs-Frobenius theory yields the large-time asymptotic solutions. The instability is found to remain, within the linear theory, of the translationally convective or shear type. No onset of rotational or vortex motion, i.e., formation of "coherent structures" occurs.
\end{abstract}

1. Introduction. The Kelvin-Helmholtz instability caused by tangential velocity shear in homogeneous fluids and plasmas is of interest in investigating a variety of space, astrophysical, and geophysical situations involving sheared plasma flows. Configurations where it is relevant include the interface between the solar wind and the magnetosphere (Sen, 1965; Southwood, 1968; Southwood, 1974; Bridge, et al., 1979; Ness, et al., 1981; $\mathrm{Pu}$ and Kivelson, 1983; Bull, 1984), coronal streamers moving through the solar wind, the boundaries between adjacent sectors in the solar wind (Parker, 1963; Sturrock and Hartle, 1966; Jokipii and Davis, 1969), the structure of the tails of comets (Dobrowolny and D'Angelo, 1972; Ershkovich, et al., 1972; Ershkovich and Chernikov, 1973; Brandt and Mendis, 1979), and the boundaries of the jets propagating from the nuclei of extragalactic double radio sources into their lobes (Turland and Scheuer, 1976; Blandford and Pringle, 1976, Begelman, et al., 1984).

Early investigations of the Kelvin-Helmholtz instability were concerned with the instability caused by a tangential velocity discontinuity or jump (or vortex sheet) in incompressible and compressible fluids and plasmas (Landau, 1944; Fejer, 1964; Sen, 1964; Miles, 1957; Gerwin, 1968).

The unmagnetized vortex sheet is found to be unstable at all wavenumbers for modes sufficiently transverse to the zero-order flow, or for modes along a flow with Mach number

Received March 15, 1994.

1991 Mathematics Subject Classification. Primary 44A10, 76E05, 76E25, 35Q10, 76J99.

Key words and phrases. Compressible Kelvin-Helmholtz instability, initial-value problem, timeasymptotics. 
less than $2 \sqrt{2}$. In the presence of a magnetic field parallel to the flow the instability of the incompressible vortex sheet is completely stabilized unless the velocity discontinuity exceeds twice the Alfvén speed. A magnetic field transverse to the flow has no effect on the instability.

Lerche (1966) emphasized the importance of considering the finite thickness of the shear layer. The linear Kelvin-Helmholtz instability of shear layers (a region of finite width over which the velocity change occurs) for flows with a subsonic velocity change was considered by Chandrasekhar (1981). An incompressible shear layer having a "hyperbolic tangent" profile was considered by Michalke (1964). He found a criterion $k L<2$ for instability, with $k$ being so short wavelength modes were stabilized for the finitewidth velocity shear. The stability characteristics of finite-width unmagnetized shear layers have been considered by several authors (Blumen, 1970; Blumen, et al., 1975; Ray, 1982; Miura and Pritchett, 1982; Roy Choudhury and Lovelace, 1984). The finite-width shear layers exhibit unstable traveling wave nodes satisfying radiation boundary conditions. These modes are absent for the unmagnetized vortex sheet, and present for the magnetized vortex sheet in a very small range of Mach numbers. The presence of the traveling wave modes means that the finite-width layer is unstable at all Mach numbers. In addition, standing wave solutions analogous to the "warping" modes which occur for the vortex sheet are also present at long wavelengths and small values of the Mach number. Magnetized shear layers described by the MHD formalism have been considered for a linear velocity profile layer (Roy Choudhury, 1986; Ray and Ershkovich, 1983; Roy Choudhury and Lovelace, 1986) with both standing and traveling wave solutions, and for a hyperbolic tangent velocity profile (Miura and Pritchett, 1982) for only standing wave modes. A magnetic field parallel to the flow is found to stabilize both classes of modes. Computer simulation studies of the Kelvin-Helmholtz instabilities of planar, magnetized shear layers (Nepveu, 1980; Tajima and Leboeuf, 1980; Miura, 1982; Pritchett and Coroniti, 1984; Miura, 1984), and of cylindrical axisymmetric jets (Norman, et al., 1982) have also been carried out. Velocity shear of zero and finite thickness have also been considered in anisotropic plasmas (Talwar, 1965; Roy Choudhury and Patel, 1985). The results are analogous to the MHD case with larger instability growth rates. Other recent work has been reviewed by Larosa and Moore (1993).

In this paper we study the general initial-value problem for the linear Kelvin-Helmholtz instability of arbitrarily compressible velocity shear layers for both the unmagnetized and magnetized cases via the use of Laplace transforms. To our knowledge, this has not been studied previously. The simplest example of the solution of the general initial-value problem for linear stability analysis of a fluid flow has been given by Case (1960a,b). Our treatment will follow that analysis to obtain the time-asymptotic solutions for this instability. The qualitative long-time features of the instability are deduced from these solutions. In particular, we find that there is no occurrence of rotational motion, i.e., the instability remains of the shear or translationally convective type.

The remainder of this paper is organized as follows. Section 2 describes the initialvalue problem, with some of the mathematical details being contained in Appendices A and $\mathrm{B}$. In $\S 3$ we obtain the long-time limit of the solutions, and discuss their implications for the behavior of the unstable modes. 
2. The initial-value problem. The fluid-dynamical equations for a compressible, inviscid, neutral fluid with an adiabatic equation of state are

$$
\begin{gathered}
\frac{\partial \rho}{\partial t}+\vec{\nabla} \cdot(\rho \vec{\nu})=0 \\
\rho \frac{d \vec{\nu}}{d t}=-\vec{\nabla} p \\
\frac{d}{d t}\left(p \rho^{-\gamma}\right)=0
\end{gathered}
$$

with $d / d t \equiv(\partial / \partial t+\vec{\nu} \cdot \vec{\nabla})$. The equilibrium we considered has a constant density $\rho$, pressure $p$, and temperature $T$.

The first-order perturbation quantities are of the form $f(x) \exp \left[i\left(k_{y} y+k_{z} z-\omega t\right)\right]$. The frequency $\omega$ is assumed to have at least a small positive imaginary part so that the solutions correspond to those of an initial-value problem. The linearization of Eq. (1) gives

$$
\begin{aligned}
i\left(k_{z} \nu_{z}-\omega\right) \rho \delta \nu_{x} & =-\delta p^{\prime}, \\
i\left(k_{z} \nu_{z}-\omega\right) \delta \rho+\rho\left(i k_{y} \delta \nu_{y}+i k_{z} \delta \nu_{z}+\delta \nu_{x}^{\prime}\right) & =0, \\
i\left(k_{z} \nu_{z}-\omega\right) \rho \delta \nu_{y} & =-i k_{y} \delta p, \\
i\left(k_{z} \nu_{z}-\omega\right) \rho \delta \nu_{z}+\rho \nu_{z}^{\prime} \delta \nu_{x} & =-i k_{z} \delta p, \\
\frac{\delta p}{p} & =\frac{\gamma \delta \rho}{\rho} .
\end{aligned}
$$

Here $\nu_{z}, p, \rho$ and the adiabatic exponent $\gamma$ are the equilibrium quantities. The prime denotes a derivative with respect to $x$.

Given the value of $\delta \rho(x, t=0), \delta \nu_{x}(x, t=0), \delta \nu_{y}(x, t=0), \delta \nu_{z}(x, t=0)$, and $\delta p(x, t=$ 0 ), we require the solution for all $t>0$. This is the initial-value problem we now solve.

Defining the Laplace transform of a variable $\delta \gamma(x, t)$ as

$$
\Gamma(x, s) \equiv \int_{0}^{\infty} e^{-s t} \delta \gamma(x, t) d t
$$

by the capital symbol, Eqs. (2) become

$$
\begin{gathered}
\frac{s P-\delta p(x, t=0)}{C_{s}^{2}}+\frac{i k_{z} \nu_{z} P}{C_{s}^{2}}+\rho\left[i k_{y} V_{y}+i k_{z} V_{z}+V_{x}^{\prime}\right]=0 \\
\rho\left[s V_{x}-\delta \nu_{x}(x, t=0)\right]+i k_{z} \nu_{z} \rho V_{x}=-P^{\prime} \\
\rho\left[s V_{z}-\delta \nu_{z}(x, t=0)\right]+i k_{z} \nu_{z} \rho V_{z}+\rho \nu_{z}^{\prime} V_{x}=-i k_{z} P .
\end{gathered}
$$

These may be combined into the composite equation

$$
\begin{aligned}
U\left[P^{\prime \prime}-\frac{2 U^{\prime} P^{\prime}}{U}+k_{z}^{2}\left(U^{2}-1\right) P\right]= & -2 \rho U^{\prime} \delta \nu_{x}(x, t=0)-U^{2} \delta p(x, t=0) \\
& +i k_{z} \rho \delta \nu_{z}(x, t=0),
\end{aligned}
$$

with the dimensionless flow velocity $U$ and adiabatic sound speed $C_{s}$ given by

$$
U \equiv \frac{k_{z} \nu_{z}-i s}{k C_{s}}
$$




$$
C_{s} \equiv\left(\frac{\gamma p}{\rho}\right)^{1 / 2} .
$$

Given the initial data, the solutions of (5) will be unique except at discrete values of $s$, which are the eigenvalues and for which $P$ satisfies the homogeneous part of (5). The solution of (5) as $s$ approaches any eigenvalue will have the solution for $P$ containing a simple pole in $s$ (for all $x$ ) (Case 1960, Kaup 1990). The solutions of the homogeneous problem with radiative boundary conditions have been considered earlier by Roy Choudhury and Lovelace (1984). Note that one may consider the non-discrete or continuum modes as the solutions of

$$
P^{\prime \prime}-\frac{2 U^{\prime} P^{\prime}}{U}+k_{z}^{2}\left(U^{2}-1\right) P=\delta(U)
$$

with $\delta$ the Kronecker or Dirac delta function.

The solution of (5) also has other singularities in $s$. These occur at the singular points of (5) and depend on $x$. These will be found to also contribute to the timeasymptotic solution. In order to consider these, it will be convenient to return to (2). Notice that the assumed time dependence $e^{-i \omega t}$ in these equations corresponds to Fourier transforming in time (Krall and Trivelpiece, 1973). We will consider (2) and later obtain the correspondence with the more standard Laplace transform in time above by setting

$$
\omega \equiv i s
$$

so that (6) yields

$$
U \equiv \frac{k_{z} \nu_{z}-i s}{k C_{s}}=\frac{k_{z} \nu_{z}-\omega}{k C_{s}} .
$$

Using Eqs. (2) (with $e^{-i \omega t}$ time dependence), we obtain the composite equation

$$
\delta p^{\prime \prime}-\frac{2 U^{\prime} \delta p^{\prime}}{U}=B^{2}\left(1-U^{2}\right) \delta p
$$

for the pressure perturbation. Here, the transverse wavenumber is

$$
B \equiv\left(k_{y}^{2}+k_{z}^{2}\right)^{1 / 2} .
$$

This equation has a regular singular point at $U=0$. The solutions near this singular point will be necessary later for the derivation of the time-asymptotic solution. This solution, and its somewhat lengthy derivation, are contained in Appendix A.

For the case of a uniform magnetic field $\vec{B}_{0}$ parallel to the direction of streaming (the $z$-direction), the ideal magnetohydrodynamic (MHD) equations for a compressible inviscid, perfectly conducting fluid are

$$
\begin{gathered}
\frac{\partial \rho}{\partial t}+\vec{\nabla} \cdot(\rho \vec{\nu})=0, \\
\rho \frac{d \vec{\nu}}{d t}=-\vec{\nabla} p+(\vec{\nabla} \times \vec{B}) \times \frac{\vec{B}}{4 \pi}, \\
\frac{\partial \vec{B}}{\partial t}=\vec{\nabla} \times(\vec{\nu} \times \vec{B}), \\
\frac{d}{d t}\left(p \rho^{-\gamma}\right)=0, \\
\vec{\nabla} \cdot \vec{B}=0,
\end{gathered}
$$


with $\frac{d}{d t}=\frac{\partial}{\partial t}+\vec{\nu} \cdot \vec{\nabla}$. The equilibrium has a flow velocity $\vec{\nu}=\hat{z} \nu_{z}(x)$, constant density $(\rho)$, pressure $(p)$, temperature $(T)$, and magnetic field $\left(\vec{B}=\hat{z} B_{0}\right)$.

These equations may be linearized using first-order perturbations of the form

$$
f(x) \exp \left[i\left(k_{y} y+k_{z} z-\omega t\right)\right]
$$

(corresponding to Fourier transforming in time and along $y$ and $z$ ) yielding (Roy Choudhury and Lovelace 1986):

$$
\begin{gathered}
i\left(k_{z} \nu_{z}-\omega\right) \delta \rho+\rho\left(i k_{y} \delta \nu_{y}+i k_{z} \delta \nu_{z}+\delta \nu_{x}^{\prime}\right)=0 \\
i\left(k_{z} \nu_{z}-\omega\right) \rho \delta \nu_{x}=-\delta p^{\prime}+\frac{B_{0}}{4 \pi}\left(i k_{z} b_{x}-b_{z}^{\prime}\right) \\
i\left(k_{z} \nu_{z}-\omega\right) \rho \delta \nu_{y}=-i k_{y} \delta p+\frac{i B_{0}}{4 \pi}\left(k_{z} b_{y}-k_{y} b_{z}\right) \\
i\left(k_{z} \nu_{z}-\omega\right) \rho \delta \nu_{z}+\rho \nu_{z}^{\prime} \delta \nu_{x}=-i k_{z} \delta p \\
\frac{\delta p}{p}=\frac{\gamma \delta \rho}{\rho} \\
i\left(k_{z} \nu_{z}-\omega\right) b_{x}=i k_{z} B_{0} \delta \nu_{x} \\
i\left(k_{z} \nu_{z}-\omega\right) b_{y}=i k_{z} B_{0} \delta \nu_{y} \\
i\left(k_{z} \nu_{z}-\omega\right) b_{z}=\nu_{z}^{\prime} b_{x}-B_{0}\left(\delta \nu_{x}^{\prime}+i k_{y} \delta \nu_{y}\right)
\end{gathered}
$$

and

$$
b_{x}^{\prime}+i k_{y} b_{y}+i k_{z} b_{z}=0 .
$$

Here, $b_{x}, b_{y}$, and $b_{z}$ are perturbation amplitudes of the magnetic field components. Equations (14) may be combined into a composite equation (Roy Choudhury and Lovelace 1986, Miura and Pritchett 1982)

$$
\left[\frac{\delta p_{*}^{\prime}}{U_{A}^{2}-1}\right]^{\prime}=K^{2}\left[\frac{\left(1+q^{2}\right) U_{A}^{2}-1-U^{2} U_{A}^{2}}{\left(U_{A}^{2}-1\right)\left\{\left(1+q^{2}\right) U_{A}^{2}-1\right\}}\right] \delta p_{*}
$$

for the total Reynold's (fluid) cum magnetic pressure perturbation

$$
\delta p_{*}=\delta p+\frac{B_{0} \delta B_{z}}{4 \pi}
$$

Here, $K \equiv\left(k_{y}^{2}+k_{z}^{2}\right)^{1 / 2}$,

$$
U_{A} \equiv\left(\frac{k C_{s}}{k_{z} \nu_{A}}\right) U
$$

with the Alfvén speed

$$
V_{A} \equiv B_{0} /(4 \pi \rho)^{1 / 2}
$$

and magnetization parameter

$$
q \equiv V_{A} / C_{S}
$$

The singular points of (15) and the solutions near those points are given in Appendix B.

Having described the Laplace transform of the general solution, we next proceed to the long-time solutions. 
3. Time-asymptotic solutions and discussion. In this section, we look at the long-time behavior of the solutions of Eq. (9). Note that

$$
u \equiv \frac{k_{z} \nu_{z}-\omega}{k C_{s}}
$$

is the negative of the angular frequency in the frame of reference of the fluid. Notice also that we used a Fourier transform in Sec. 2 (all perturbation quantities were of the form $\left.e^{-i \omega t}\right)$. Hence, to consider the correspondence with the more standard Laplace transform in time, we make the replacement (8a) or, in the moving frame,

$$
u \equiv\left(\frac{k_{z} \nu_{z}-i s}{k C_{s}}\right)=-\frac{i}{k C_{s}}\left(s+i k_{z} \nu_{z}\right)
$$

Thus, small values of $u$ correspond to small $s$ and, hence, to long-time asymptotic solutions. Similarly, around a singularity at $u=\alpha$ the dominant terms in powers of $(u-\alpha)$ around the singularity govern the long-time asymptotic behavior from that solution.

From Eqs. (A11) and (A12), the general solution of the fluid-dynamical pressureperturbation equation (9) around the singularity $u=0$ is

$$
\delta p=\alpha_{0}\left[1-\frac{B_{*}^{2} u^{2}}{2}+O\left(u^{4}\right)\right]+\alpha_{2}\left[1+\frac{B_{*}^{2} u^{2}}{10}+O\left(u^{4}\right)\right]
$$

Using (2) and (20a) yields

$$
\begin{aligned}
& \delta \nu_{x}(s)=\text { constant } \\
& \delta \nu_{y}(s)=\text { constant } / r \\
& \delta \nu_{z}(s)=\text { constant } / r
\end{aligned}
$$

where

$$
r \equiv s+i k_{z} \nu_{z}(x)
$$

There are two basic contributions to the solution of the initial-value problem: (i) contours around the eigenvalues and (ii) for any fixed value of $x$, there will be a regular singular point of the differential system (9).

The contribution from the eigenvalues will be a growing (global) eigenmode with a frequency of $\operatorname{Im}\left(s_{j}\right)$ and a growth rate of $\operatorname{Re}\left(s_{j}\right)$, where $s_{j}$ is the eigenvalue. This contribution has been very well described in the literature (Roy Choudhury and Lovelace, 1984, 1986; Miura and Pritchett, 1982).

On the other hand, the pole singularities can also give a contribution. For example, (20c) has a pole of order 1 about $r=0$. Such a pole would give a nonzero contribution.

Using the identity (19), and noting that the dominant long-time asymptotic behavior is obtained from the dominant small- $u$ behavior in the Laplace transformed domain, we may obtain the time-asymptotic behavior of the fluid-dynamical velocity shear layer by 
inverse-Laplace transforming (20). Letting $\alpha(s, x)$ be analytic in $s$ near $r=0$, one may straightforwardly show that for large times (Kaup, 1990)

$$
\begin{gathered}
\frac{1}{2 \pi i} \int_{R-i^{\infty}}^{R+i^{\infty}} \frac{\alpha(s, x)}{r^{2}(s, x)} e^{s t} d s=t e^{-i k_{z} \nu_{z}(x) t} \alpha\left(-i k_{z} \nu_{z}(x), x\right) \\
\frac{1}{2 \pi i} \int_{R-i^{\infty}}^{R+i^{\infty}} \frac{\alpha(s, x)}{r(s, x)} e^{s t} d s=e^{-i k_{z} \nu_{z}(x) t} \alpha\left(-i k_{z} \nu_{z}(x), x\right) \\
\frac{1}{2 \pi i} \int_{R-i^{\infty}}^{R+i^{\infty}} \alpha(\beta, x) e^{s t}(\ln r) d s \rightarrow 0
\end{gathered}
$$

where $R$ is chosen so that the contour is to the right of all singularities in the complex $s$-plane.

Using standard Laplace inversion formulae, (20a) yields

$$
\delta p(t) \sim \delta(t)
$$

Next, using (20b-d) and (22) the long-time asymptotic behavior of the velocity perturbations is

$$
\begin{aligned}
& \delta \nu_{y}(t) \sim e^{-i k_{z} \nu_{z}(x) t} e^{i\left(k_{y} y+k_{z} z\right)} \\
& \delta \nu_{z}(t) \sim e^{-i k_{z} \nu_{z}(x) t} e^{i\left(k_{y} y+k_{z} z\right)} \\
& \delta \nu_{x}(t) \sim \delta(t)
\end{aligned}
$$

where the $y$ and $z$ dependences have been included. The time-asymptotic behavior of the perturbations is thus fairly simple. The pressure and $x$-velocity perturbations $\delta p$ and $\delta \nu_{x}$ die away to zero. On the other hand, the $y$ and $z$-velocity perturbations approach a constant amplitude and propagate in the $y$ and $z$ directions. In the $z$-direction, they correspond to traveling waves propagating with the background flow, i.e., at the phase speed $\nu_{z}(x)$. In the $y$-direction, they propagate at a speed $k_{z} \nu_{z}(x) / k_{y}$ at each value of $x$.

Similarly, using (B9) and (B10), the general solution of the magnetic pressure-perturbation equation (15) around the singularity $u_{A}=1$ is

$$
\begin{aligned}
\delta p_{*}=c_{1}\left[a_{0}\right. & \left.+a_{2}\left(u_{A}-1\right)^{2}+O\left(u_{A}-1\right)^{4}\right] \\
& +c_{2} a_{0}\left(u_{A}-1\right)^{2}+O\left(u_{A}-1\right)^{4}
\end{aligned}
$$

and using (B21) and (B31), the solution of (15) around the singularity $u_{A}=1 / \sqrt{1+q^{2}}$ is

$$
\begin{gathered}
\delta p_{*}=d_{1}\left[a_{0} z+\left(\frac{\tau+\sigma}{2}\right) a_{0} z^{2}+O\left(z^{3}\right)\right] \\
+d_{2}\left[c_{0}+c_{1} z+O\left(z^{2}\right)\right]
\end{gathered}
$$

where $z \equiv u_{A}-\frac{1}{\sqrt{1+q^{2}}}$. 
Proceeding analogously for the magnetized case, the inverse Laplace transform of (24) (around $u_{A}=1$ ) yields

$$
\delta p_{*} \sim \delta(t)
$$

Using the linearized equations (14) of Sec. 2 in conjunction with (24) and (22), one obtains the following large-time behaviors of some of the other physical variables (from the singularity at $u_{A}=1$ ):

$$
\begin{gathered}
b_{x}(t) \sim \delta(t), \\
b_{y}(t) \sim e^{-i k_{z} \nu_{z}(x) t} e^{i\left(k_{y} y+k_{z} z\right)}, \\
b_{z}(t) \sim \delta(t), \\
\delta \nu_{y}(t) \sim \alpha_{1} e^{-i k_{z} \nu_{z} t}+\beta_{1} \delta(t), \\
\delta p(t) \sim \delta(t) .
\end{gathered}
$$

Analogously, using (25), the linearized equations of Sec. 2, and (22) yield the following time-asymptotic behaviors caused by the singularity $u_{A}=1 / \sqrt{1+q^{2}}$ of some of the relevant physical variables:

$$
\begin{gathered}
\delta p_{*}(t) \sim \delta(t), \\
b_{x}(t) \sim \delta(t), \\
b_{y}(t) \sim \delta(t), \\
b_{z}(t) \sim e^{-i k_{z} \nu_{z}(x) t}, \\
\delta \nu_{y}(t) \sim \alpha_{2} e^{-i k_{z} \nu_{z}(x) t}+\beta_{2} \delta(t), \\
\delta p(t) \sim \alpha_{3} e^{-i k_{z} \nu_{z}(x) t}+\beta_{3} \delta(t) .
\end{gathered}
$$

From (26) and (27), it is clear that, for the magnetized case, the time-asymptotic behavior is similar to that found for the fluid-dynamical case. Some of the perturbations of the physical variables die away at large times, while others approach traveling waves of constant amplitude propagating in the $z$-direction with the background flow at speed $\nu_{z}(x)$, and propagating in the $y$-direction at phase-speed $k_{z} \nu_{z}(x) / k_{y}$.

In particular, the results obtained above from the Laplace transform solution of the initial-value problem for the linear Kelvin-Helmholtz instability establish that the linear instability remains essentially a shear-instability, with translations occurring in $y$ and $z$. No rotational motion, or vortex or "coherent-structure" formation, in the regions of layers of fluid near the singularities, occurs. This is in contrast, for example, to the rotational vortex motion generated near the so-called "magnetron" singularity for shear-flows in non-neutral pure-electron plasmas (Kaup, 1990). The reason for the absence of such coherent-structure formation in our problem is not difficult to establish mathematically. The singularities in our case occurred at $u=0, U_{A}= \pm 1, \pm\left(1+q^{2}\right)^{-1 / 2}$, and were thus real. Imaginary singularities $u= \pm i \Omega$ are necessary for the establishment of vortex motion with angular frequency $\pm \Omega$ of any physical variable at large-time. Physically, too, the complex interaction of the electromagnetic fields with the non-neutral fluid 
(electron plasma), leading to the formation of rotating vortices, is absent for our neutral fluids. The analysis above demonstrates that this clearly precludes the onset of vortex motion in the long-time behavior of the supersonic Kelvin-Helmholtz instability, at least while the perturbation amplitudes are small enough for the linear theory to remain valid. In the nonlinear regime, it is well known that this instability leads to coherent vortex structures (Brown and Roshko, 1974; Winant and Browand, 1974; Aref and Siggia, 1981; Norman et al, 1982; Aref and Tryggvason, 1984). Note that the contribution from the eigenvalues, which have been considered earlier by normal mode analysis (Roy Choudhury and Lovelace, 1984; Miura and Pritchett, 1982) gives growing or unstable solutions which eventually become too large to be described by the linear theory.

Appendix A. Singularity analysis of pressure perturbation equation for supersonic fluid-dynamical shear layers. The equation for the pressure perturbation was derived in Sec. 2 and it has the form

$$
\delta p^{\prime \prime}-\frac{2 u^{\prime}}{u} \delta p^{\prime}=B^{2}\left(1-u^{2}\right) \delta p
$$

where

$$
'=\frac{d}{d \bar{x}}, \quad u=2 A \bar{x}-w .
$$

Here, we assume a linear velocity profile (Roy Choudhury and Lovelace, 1984; Ray, 1982). By taking the derivative of $u$ with respect to $\bar{x}$, we get $\frac{d u}{d \bar{x}}=2 A$. Replacing $d \bar{x}$ by $\frac{d u}{2 A}$ in (A1), Eq. (A1) takes the following form:

$$
\frac{d}{d u^{2}}(\delta p)-\frac{2}{u} \frac{d}{d u}(\delta p)+\left(\frac{B}{2 A}\right)^{2}\left(u^{2}-1\right) \delta p=0
$$

Letting $\frac{B}{2 A}=B_{*}$ and $\delta p=p$, our differential equation takes the form

$$
\frac{d^{2} p}{d u^{2}}-\frac{2 d p}{u d u}+B_{*}^{2}\left(u^{2}-1\right) p=0
$$

This differential equation has a regular singular point at $u=0$. Rewriting (A2) in the form

$$
\frac{d^{2} p}{d u^{2}}+\frac{p(u)}{u} \frac{d p}{d u}+\frac{q(u)}{u^{2}} p=0
$$

we have $p(u)=-2, q(u)=B_{*}^{2}\left(u^{2}-1\right) u^{2}$. The functions $q(u)$ and $p(u)$ are analytic at $u=0$, where (A3) has a regular singular point. Thus, we may expand $p(u)$ and $q(u)$ in Taylor Series about that point:

$$
p(u)=\sum_{n=0}^{\infty} p_{n} u^{n}=-2, \quad q(u)=\sum_{n=0}^{\infty} q_{n} u^{n}=B_{*}^{2}\left(u^{4}-u^{2}\right) .
$$

From $\sum_{n=0}^{\infty} p_{n} u^{n}=-2$, we get $p_{0}+p_{1} u+p_{2} u^{2}=-2$, which implies

$$
p_{0}=-2, \quad p_{1}=p_{2}=p_{3}=\cdots=0 .
$$


From $\sum_{n=0}^{\infty} q_{n} u^{n}=B_{*}^{2}\left(u^{4}-u^{2}\right)$, we get $q_{0}+q_{1} u+q_{2} u^{2}+\cdots=-B_{*}^{2} u^{2}+B_{*}^{2} u^{4}$ which implies

$$
q_{0}=q_{1}=q_{3}=q_{5}=q_{6}=\cdots=0, \quad q_{2}=-B_{*}^{2}, \quad q_{4}=B_{*}^{2} .
$$

We use the Fuchs-Frobenius method (Ince, 1956; Bender and Orszag, 1978) to find the solution of (A3) about $u=0$. Let $p(u, \alpha)=\sum_{n=0}^{\infty} a_{n} u^{n+\alpha}$, where $\alpha$ is the indicial exponent to be determined. By substituting the Taylor expansion of $p(u, \alpha)$ in (A3), we get

$$
\begin{gathered}
\frac{d p}{d u}=\sum_{n=0}^{\infty} a_{n}(n+\alpha) u^{n+\alpha-1}, \quad \frac{d^{2} p}{d u^{2}}=\sum_{n=0}^{\infty} a_{n}(n+\alpha)(n+\alpha-1) u^{n+\alpha-2} \\
\sum_{n=0}^{\infty} a_{n}(n+\alpha)(n+\alpha-1) u^{n+\alpha-2}-2 \sum_{n=0}^{\infty} a_{n}(n+\alpha) u^{n+\alpha-2}+B_{*}^{2}\left(u^{2}-1\right) \sum_{n=0}^{\infty} a_{n} u^{n+\alpha}=0 \\
\left.\sum_{n=0}^{\infty} a_{n}(n+\alpha)(n+\alpha-3) u^{n+\alpha-2}+B_{*}^{2} \sum_{n=0}^{\infty} a_{n} u^{n+\alpha+2}-B_{*}^{2} \sum_{n=0}^{\infty} \alpha_{n} u^{n+\alpha}=0, \quad \text { (A } 7\right) \\
\sum_{n=0}^{\infty} a_{n}(n+\alpha)(n+\alpha-3) u^{n+\alpha-2}=a_{0} \alpha(\alpha-3) u^{\alpha-2}+a_{1}(1+\alpha)(\alpha-2) u^{\alpha-1} \\
+a_{2}(2+\alpha)(\alpha-1) u^{\alpha}+a_{3}(3+\alpha) \alpha u^{\alpha+1} \\
+\sum_{n=0}^{\infty} a_{n+4}(n+4+\alpha)(n+\alpha+1) u^{n+\alpha+2} \\
\sum_{n=0}^{\infty} a_{n} u^{n+\alpha}=a_{0} u^{\alpha}+a_{1} u^{1+\alpha}+\sum_{n=0}^{\infty} a_{n+2} u^{n+\alpha+2} .
\end{gathered}
$$

Substitution of (A8), (A9) into (A7) and rearrangement of terms yields

$$
\begin{aligned}
& a_{0} \alpha(\alpha-3) u^{\alpha-2}+a_{1}(\alpha+1)(\alpha-2) u^{\alpha-1}+\left[a_{2}(\alpha+2)(\alpha-1)-B_{*}^{2} a_{0}\right] u^{\alpha} \\
& \quad+\left[a_{3}(\alpha+3) \alpha-B_{*}^{2} a_{1}\right] u^{\alpha+1} \\
& \quad+\sum_{n=0}^{\infty}\left[a_{n+4}(n+4+\alpha)(n+\alpha+1)-B_{*}^{2}\left(a_{n+2}-a_{n}\right)\right] u^{n+\alpha+2}=0 .
\end{aligned}
$$

By setting the coefficients of $u^{\alpha-2}, u^{\alpha-1}, u^{\alpha}, u^{\alpha+1}, u^{n+\alpha+2}$ equal to zero we can find the values of $\alpha$ and we can derive the recursion relation

$$
\begin{gathered}
a_{0} \alpha(\alpha-3)=0 \quad: u^{\alpha-2}, \\
a_{1}(\alpha+1)(\alpha-2)=0 \quad: u^{\alpha-1}, \\
a_{2}(\alpha+2)(\alpha-1)-B_{*}^{2} a_{0}=0 \quad: u^{\alpha}, \\
a_{3}(\alpha+3) \alpha-B_{*}^{2} a_{1}=0 \quad: u^{\alpha+1}, \\
a_{n+4}(n+4+\alpha)(n+\alpha+1)-B_{*}^{2}\left(a_{n+2}-a_{n}\right)=0 \quad: u^{n+\alpha+2} .
\end{gathered}
$$

By assumption $a_{0} \neq 0$, therefore $\alpha(\alpha-3)=0$, which implies $\alpha_{1}=3, \alpha_{2}=0$. From $a_{1}(\alpha+1)(\alpha-2)=0$, we conclude that $a_{1}=0$ since $(\alpha+1)(\alpha-2) \neq 0$ for $\alpha=0, \alpha=3$. 
From $a_{2}(\alpha+2)(\alpha-1)-B_{*}^{2} a_{0}=0$, we conclude $a_{2}=\frac{B_{*}^{2}}{(\alpha+2)(\alpha-1)} a_{0}$ in the equation $a_{3}(\alpha+3) \alpha-B_{*}^{2} a_{1}=0$. Since $a_{1}=0, a_{3}(\alpha+3) \alpha=0$, there are two cases to be considered.

Case 1) $\alpha=0$; then $a_{3}$ is arbitrary; and

Case 2) $\alpha=3$; then $a_{3}=0$.

The recursion relationship can be written as

$$
a_{n+4}=\frac{B_{*}^{2}\left(a_{n-2}-a_{n}\right)}{(n+\alpha+1)(n+\alpha+4)}, \quad n=0,1,2,3, \ldots
$$

We discuss the existence of linearly independent solutions in Frobenius form. So we check both sides of the following equality (Bender and Orszag, 1978):

$$
0 \cdot a_{N}=-\sum_{k=0}^{N-1}\left[(\alpha+k) p_{N-k}+q_{N-k}\right] a_{k}
$$

where $N=\alpha_{1}-\alpha_{3}=3, \alpha=\alpha_{2}=0$;

$$
0 \cdot a_{3}=-\sum_{k=0}^{2}\left[k p_{3-k}+q_{3-k}\right] a_{k}=-\left[0+q_{3}\right] a_{0}-\left[p_{2}+q_{2}\right] a_{1}-\left[2 p_{1}+q_{1}\right] a_{2}
$$

but from (A5), (A6) we have $q_{3}=0, p_{2}=0, q_{2}=-B_{*}^{2}, p_{1}=q_{1}=0, a_{1}=0$. Therefore, (A10) would read

$$
0 \cdot a_{3}=-[0+0] a_{0}-\left[0-B_{*}^{2}\right] 0-[0+0] a_{2}=0
$$

or $a_{3}$ is arbitrary. There are thus two linearly independent solutions of (A3) around the regular singular point $u=0$ in Frobenius form (Bender and Orszag, 1978).

Consider the various cases separately:

$$
\begin{aligned}
\alpha & =0 \\
a_{0} & \neq 0 \text { by assumption, } \\
a_{1} & =0 \\
a_{2} & =\frac{B_{*}^{2} a_{0}}{(\alpha+2)(\alpha-1)} \text { when } \alpha=0, \quad a_{2}=\frac{-B_{*}^{2}}{2} a_{0},
\end{aligned}
$$

$a_{3}$ is arbitrary, $\quad$ recursion relationship $a_{n+4}=\frac{B_{*}^{2}\left(a_{n+2}-a_{n}\right)}{(n+\alpha+1)(n+\alpha+4)}$.

For this case, $\alpha=0$; so we have

$$
a_{n+4}=\frac{B_{*}^{2}\left(a_{n+2}-a_{n}\right)}{(n+1)(n+4)} .
$$


Setting $n=0,1,2, \ldots$, we can calculate $a_{4}, a_{5}, a_{6}, \ldots$ :

$$
\begin{aligned}
n=0: a_{4} & =\frac{B_{*}^{2}\left(a_{2}-a_{0}\right)}{4} \text { but } a_{2}=\frac{-B_{*}^{2}}{2} a_{0}, \text { and after substitution we have } \\
a_{4} & =\frac{B_{*}^{2}}{4}\left(\frac{-B_{*}^{2}}{2} a_{0}-a_{0}\right)=\frac{-B_{*}^{2}}{8}\left(B_{*}^{2}+2\right) a_{0} \\
n=1: a_{5} & =\frac{B_{*}^{2}\left(a_{3}-a_{1}\right)}{10} \text { but } a_{1}=0, \text { and } a_{3} \text { is arbitrary, } \\
a_{5}= & \frac{B_{*}^{2}}{10} a_{3}, \\
n=2: a_{6} & =\frac{B_{*}^{2}\left(a_{4}-a_{2}\right)}{3(6)}=\frac{B_{*}^{2}}{18}\left(\frac{-B_{*}^{2}}{8}\left(B_{*}^{2}+2\right) a_{0}+\frac{B_{*}^{2}}{2} a_{0}\right) \\
& =\frac{B_{*}^{4}}{144}\left(-B_{*}^{2}-2+4\right) a_{0}=\frac{B_{*}^{4}}{144}\left(2-B_{*}^{2}\right) a_{0}, \\
n=3: a_{7} & =\frac{B_{*}^{2}\left(a_{5}-a_{3}\right)}{28}=\frac{B_{*}^{2}}{28}\left(\frac{B_{*}^{2}}{10} a_{3}-a_{3}\right)=\frac{B_{*}^{2}}{280}\left(B_{*}^{2}-10\right) a_{3}, \\
n=4: a_{8}= & \frac{B_{*}^{2}\left(a_{6}-a_{4}\right)}{40}=\frac{B_{*}^{2}}{40}\left(\frac{B_{*}^{4}}{144}\left(2-B_{*}^{2}\right) a_{0}+\frac{B_{*}^{4}}{8}\left(B_{*}^{2}+2\right) a_{0}\right) \\
a_{8} & =\frac{B_{*}^{4}}{5760}\left(-B_{*}^{4}+20 B_{*}^{2}+36\right) a_{0} .
\end{aligned}
$$

For $\alpha=0$, we have a solution of Frobenius form:

$$
p(u, 0)=\sum_{n=0}^{\infty} a_{n} u^{n}=a_{0}+a_{1} u+a_{2} u^{2}+a_{3} u^{3}+a_{4} u^{4}+a_{5} u^{5}+\cdots
$$

Substituting the values of $a_{1}, a_{2}, a_{3}$, the solution corresponding to $\alpha=0$ takes the form

$$
\begin{aligned}
p(u, 0)= & a_{0}\left[\left(1-\frac{B_{*}^{2}}{2} u^{2}\right)-\frac{B_{*}^{2}}{8}\left(B_{*}^{2}+2\right) u^{4}+\frac{B_{*}^{4}}{144}\left(2-B_{*}^{2}\right) u^{6}\right. \\
& \left.+\frac{B_{*}^{4}}{5760}\left(-B_{*}^{4}+20 B_{*}^{2}+36\right) a_{0} u^{8}+\cdots\right] \\
& +\left[a_{3} u^{3}+\frac{B_{*}^{2}}{10} u^{5}+\frac{B_{*}^{2}}{280}\left(B_{*}^{2}-10\right) u^{7}+\cdots\right] .
\end{aligned}
$$

We consider the case when $\alpha=3$ next:

$$
\begin{aligned}
p(u, 3) & =\sum_{n=0}^{\infty} a_{n} u^{n+3} ; \text { to find } a_{n} \text { 's we use the recursion relationship; } \\
a_{n+4} & =\frac{B_{*}^{2}\left(a_{n+2}-a_{n}\right)}{(n+\alpha+1)(n+\alpha+4)} . \text { Setting } \alpha=3 \text { we have } \\
a_{n+4} & =\frac{B_{*}^{2}\left(a_{n+2}-a_{n}\right)}{(n+4)(n+7)}, \text { where } a_{1}=0 \text { and } a_{3}=0, a_{0} \neq 0 . \text { Also, for } \alpha=3 \\
\frac{B_{*}^{2} a_{0}}{(\alpha+2)(\alpha-1)} & =\frac{B_{*}^{2} a_{0}}{10} .
\end{aligned}
$$


We also have

$$
\begin{aligned}
& n=0: a_{4}=\frac{B_{*}^{2}\left(a_{2}-a_{0}\right)}{28}=\frac{B_{*}^{2}}{28}\left(\frac{B_{*}^{2} a_{0}}{10}-a_{0}\right)=\frac{B_{*}^{2}}{280}\left(B_{*}^{2}-10\right) a_{0}, \\
& n=1: a_{5}=\frac{B_{*}^{2}\left(a_{3}-a_{1}\right)}{40}=\frac{B_{*}^{2}}{40}\left(0-a_{1}\right)=-\frac{B_{*}^{2}}{40} a_{1}, \\
& n=2: a_{6}=\frac{B_{*}^{2}\left(a_{4}-a_{2}\right)}{6(9)}=\frac{B_{*}^{2}}{54}\left(\frac{B_{*}^{2}\left(B_{*}^{2}-10\right)}{280}-\frac{B_{*}^{2}}{10}\right) a_{0}=\frac{B_{*}^{4}}{15120}\left(B_{*}^{2}-38\right) a_{0}, \\
& n=3: a_{7}=\frac{B_{*}^{2}\left(a_{5}-a_{3}\right)}{70}=\frac{B_{*}^{2}}{70}\left(\frac{-B_{*}^{2}}{40} a_{1}-0\right)=\frac{-B_{*}^{4}}{2800} a_{1}, \\
& n=4: a_{8}=\frac{B_{*}^{2}\left(a_{6}-a_{4}\right)}{88}=\frac{B_{*}^{2}}{88}\left(\frac{B_{*}^{4}}{15120}\left(B_{*}^{2}-38\right) a_{0}-\frac{B_{*}^{2}}{280}\left(B_{*}^{2}-10\right) a_{0}\right), \text { or } \\
& a_{8}=\frac{B_{*}^{4}}{1330560}\left(B_{*}^{4}-92 B_{*}^{2}+540\right) a_{0} .
\end{aligned}
$$

The Frobenius solution for the case when $\alpha=3$ has the form

$$
p(u, 3)=a_{0}+a_{1} u+a_{2} u^{2}+a_{3} u^{3}+a_{4} u^{4}+a_{5} u^{5}+\cdots .
$$

Substituting the values of $a_{0}, a_{1}, a_{2}, a_{3}, a_{4}, a_{5}$, etc., in the above expression and taking into account that $a_{0} \neq 0, a_{1}=0, a_{3}=0$, the second linearly independent solution of Frobenius type takes the following form (Bender and Orszag, 1978):

$$
\begin{gathered}
p(u, 3)=\left(1+\frac{B_{*}^{2}}{10} u^{2}+\frac{B_{*}^{2}}{280}\left(B_{*}^{2}-10\right) u^{4}+\frac{B_{*}^{4}}{15120}\left(B_{*}^{2}-38\right) u^{6}\right. \\
\left.+\frac{B_{*}^{4}}{1330560}\left(B_{*}^{4}-92 B_{*}^{2}+540\right) u^{8}+\cdots\right) a_{0} \\
+\left(-\frac{B_{*}^{2}}{40} u^{5}-\frac{B_{*}^{4}}{2800} u^{7}+\frac{B_{*}^{4}}{302400}\left(-B_{*}^{2}+70\right) u^{9}+\cdots\right) a_{1}
\end{gathered}
$$

The general solution to (A3) is a linear combination of $p(u, 3)$ and $p(u, 0)$.

\section{Appendix B. Singularity analysis for magnetohydrodynamic pressure equa-} tion. It was shown in Sec. 2 that the total pressure perturbation corresponding to the magnetized case satisfies the following equation:

$$
\left[\frac{\left(\delta p_{*}\right)^{\prime}}{u_{A}^{2}-1}\right]^{\prime}=K^{2}\left[\frac{\left(1+q^{2}\right) u_{A}^{2}-1-u^{2} u_{A}^{2}}{\left(u_{A}^{2}-1\right)\left(\left(1-q^{2}\right) u_{A}^{2}-1\right)}\right] \delta p_{*} .
$$

Rewrite the above equation in the following form:

$$
\left(\delta p_{*}\right)^{\prime \prime}-\frac{2 u_{A}^{\prime} u_{A}}{\left(u_{A}^{2}-1\right)}\left(\delta p_{*}\right)^{\prime}=K^{2}\left[\frac{\left(1+q^{2}\right) u_{A}^{2}-1-u^{2} u_{A}^{2}}{\left(\left(1+q^{2}\right) u_{A}^{2}-1\right)}\right] \delta p_{*} .
$$

The singular points of this equation occur where $u_{A}^{2}-1=0$ and where $\left(1+q^{2}\right) u_{A}^{2}-1=0$. 
The vanishing of the factor $\left(u_{A}^{2}-1\right)$ can be written as the following:

$$
u_{A}^{2}-1=0, \quad u_{A}=\frac{k}{k_{z}} \frac{c_{s}}{v_{A}} u=\frac{k c_{s}}{k_{z} v_{A}}\left(\frac{k_{z} v_{z}}{k c_{s}}-\frac{\omega}{k c_{s}}\right)=\frac{k_{z} v_{z}-\omega}{k_{z} v_{A}} .
$$

Upon substituting in $u_{A}^{2}-1=0$ we get $\left(\omega-k_{z} v_{z}\right)^{2}=\left(k_{z} v_{A}\right)^{2}$, which is the local dispersion relation for shear Alfvén waves which propagate relative to the fluid at the speed $v_{A}$ parallel to the magnetic field. This is called the Alfvén singularity in magnetohydrodynamics (MHD). The vanishing of the factor $\left[\left(1+q^{2}\right) u_{A}^{2}-1\right]$ corresponds to wave propagation relative to the fluid (in the $z$-direction) at the slow magnetosonic wave front propagation speed $v_{0} \equiv v_{A}\left(1+q^{2}\right)^{-1 / 2}$ where $q=v_{A} / c_{s}$ (magnetization parameter).

Equation (B1) has two other critical points, corresponding to the vanishing of the numerator of the right-hand side of (B1). The vanishing of the factor $\left[\left(1+q^{2}\right) u_{A}^{2}-1-\right.$ $u^{2} u_{A}^{2}$ ] occurs at two values of $u_{A}^{2}$ which correspond to the fast and the slow magnetosonic waves that propagate relative to the fluid in the $z$-direction at phase and group velocities given by this relation. The vanishing of the factor $u_{A}^{2}-1=0$ corresponds to $u_{A}= \pm 1$, using the relationship $u_{A}=M_{A} x-W_{a}$ where $M_{A}=2 v_{z m} / v_{A}, v_{z m}=v_{z}(x>1 / 2)$, and $v_{z}(x)$ is the equilibrium flow velocity in the $z$-direction. $M_{A}$ is the reduced Mach number defined previously. Differentiating the relationship $u_{A}=M_{A} x-W_{A}$, we have $d u_{A} / d x=M_{A}$ and

$$
\begin{gathered}
\left(\delta p_{x}\right)^{\prime}=\frac{d}{d x}\left(\delta p_{*}\right)=\frac{d\left(\delta p_{*}\right)}{d u_{A}} \frac{d u_{A}}{d x}=M_{A} \frac{d\left(\delta p_{*}\right)}{d u_{A}} \\
\left(\delta p_{x}\right)^{\prime \prime}=\frac{d}{d x}\left(\delta p_{*}\right)^{\prime}=\frac{d\left(\delta p_{*}\right)^{\prime}}{d u_{A}} \frac{d u_{A}}{d x}=M_{A} \frac{d^{2}\left(\delta p_{*}\right)}{d u_{A}^{2}}\left(M_{A}\right)=M_{A}^{2} \frac{d^{2}\left(\delta p_{*}\right)}{d u_{A}^{2}}
\end{gathered}
$$

Upon substituting these in (B1), this differential equation reduces to

$$
M_{A}^{2} \frac{d^{2}\left(\delta p_{*}\right)}{d u_{A}^{2}}-2 M_{A} \frac{u_{A}}{u_{A}^{2}-1} \cdot M_{A} \frac{d\left(\delta p_{*}\right)}{d u_{A}}=k^{2}\left[\frac{\left(1+q^{2}\right) u_{A}^{2}-1-u^{2} u_{A}^{2}}{\left(1+q^{2}\right) u_{A}^{2}-1}\right] \delta p_{*} .
$$

In the vicinity of $u_{A}=1, u_{a}+1 \approx 2$, setting $u_{A}=1$ in the right-hand side of $(\mathrm{B} 2)$ we further simplify (B2) to

$$
M_{A}^{2} \frac{d^{2}\left(\delta p_{*}\right)}{d u_{A}^{2}}-2 M_{A} \frac{u_{A}}{u_{A}^{2}-1} \cdot M_{A} \frac{d\left(\delta p_{*}\right)}{d u_{A}}=k^{2}\left[\frac{\left(1+q^{2}\right) u_{A}^{2}-1-u^{2} u_{A}^{2}}{\left(1+q^{2}\right) u_{A}^{2}-1}\right] \delta p_{*} .
$$

Here

$$
u_{A}=\frac{k}{k_{z}}\left(\frac{c_{s}}{v_{A}}\right) u
$$

where $q=v_{A} / c_{s}$ (magnetization factor), $q \neq 0$. Setting $u_{A}=1$, we get

$$
\frac{u}{q}=\frac{k_{z}}{k}, \quad 1-\left(\frac{u}{q}\right)^{2}=1-\frac{k_{z}^{2}}{k^{2}}=\frac{k^{2}-k_{z}^{2}}{k^{2}}=\frac{k_{y}^{2}}{k^{2}}
$$

Upon substitution in the right-hand side of the above equation, (B3) simplifies to

$$
\frac{d^{2}\left(\delta p_{*}\right)}{d u_{A}^{2}}-\frac{1}{u_{A}-1} \frac{d\left(\delta p_{*}\right)}{d u_{A}}=\frac{k_{y}^{2}}{M_{A}^{2}} \delta p_{*} .
$$


We further make the change of variables $z=u_{A}-1$. Then $d u_{A}=d z$ and (B4) takes the final form

$$
\frac{d^{2}\left(\delta p_{*}\right)}{d z^{2}}-\frac{1}{z} \frac{d\left(\delta p_{*}\right)}{d z}-\beta^{2} \delta p_{*}=0
$$

Equation (B5) has a regular singular point at $z=0$. We assume a Frobenius solution of the form $\delta p_{*}=\sum_{n=0}^{\infty} a_{n} z^{n+\alpha}$ where $\alpha$ is the indicial exponent, which is to be determined. Also, substituting

$$
\frac{d\left(\delta p_{*}\right)}{d z}=\sum_{n=0}^{\infty}(n+\alpha) a_{n} z^{n+\alpha-1}, \quad \frac{d^{2}\left(\delta p_{*}\right)}{d z^{2}}=\sum_{n=0}^{\infty}(n+\alpha)(n+\alpha-1) a_{n} z^{n+\alpha-2}
$$

into (B5), we have

$$
\sum_{n=0}^{\infty}(n+\alpha)(n+\alpha-1) a_{n} z^{n+\alpha-2}-\sum_{n=0}^{\infty}(n+\alpha) a_{n} z^{n+\alpha-2}-\beta^{2} \sum_{n=0}^{\infty} a_{n} z^{n+\alpha}=0 .
$$

By putting the first two terms under a common summation we get

$$
\sum_{n=0}^{\infty}(n+\alpha)(n+\alpha-2) a_{n} z^{n+\alpha-2}-\beta^{2} \sum_{n=0}^{\infty} a_{n} z^{n+\alpha}=0 .
$$

The first term further can be written in the form

$$
\begin{aligned}
\sum_{n=0}^{\infty}(n+\alpha)(n+\alpha-2) a_{n} z^{n+\alpha-2}= & \alpha(\alpha-2) a_{0} z^{\alpha-2}+(\alpha+1)(\alpha-1) a_{1} z^{\alpha-1} \\
& +\sum_{n=0}^{\infty}(n+\alpha)(n+\alpha+2) a_{n+2} z^{n+\alpha} .
\end{aligned}
$$

Upon substituting in (B6) we can calculate the indicial exponent $\alpha$ as follows:

$$
\begin{gathered}
\alpha(\alpha-2) a_{0} z^{\alpha-2}+(\alpha+1)(\alpha-1) a_{1} z^{\alpha-1}+\sum_{n=0}^{\infty}(n+\alpha)(n+\alpha+2) a_{n+2} z^{n+\alpha} \\
-\beta^{2} \sum_{n=0}^{\infty} a_{n} z^{n+\alpha}=0 .
\end{gathered}
$$

Putting the last two terms under a common summation, we further get

$$
\begin{aligned}
& \alpha(\alpha-2) a_{0} z^{\alpha-2}+\left(\alpha^{2}-1\right) a_{1} z^{\alpha-1} \\
& \quad+\sum_{n=0}^{\infty}\left((n+\alpha)(n+\alpha+2) a_{n+2}-\beta^{2} a_{n}\right) z^{n+\alpha}=0 .
\end{aligned}
$$

Setting coefficients of $z^{\alpha-2}, z^{\alpha-1}, z^{n+\alpha}$ equal to zero we can find the $a_{i}$ 's and derive the recursion relationship. The assumption is that $a_{0} \neq 0$. From $\alpha(\alpha-2) a_{0}=0$ and $a_{0} \neq 0$ 
we conclude $\alpha_{1}=0, \alpha_{2}=2$. The vanishing of the term $\left(\alpha^{2}-1\right) a_{1}=0$ implies that $a_{1}=0$. By setting $(n+\alpha)(n+\alpha+2) a_{n+2}-\beta^{2} a_{n}=0$, we derive the indicial equation

$$
\alpha_{n+2}=\frac{\beta^{2}}{(n+\alpha)(n+\alpha+2)} a_{n}
$$

Two cases are to be considered: $\alpha=0$ and $\alpha=2$. When $\alpha=0$, the recursion relation takes the form $a_{n+2}=\frac{\beta^{2}}{n(n+2)} a_{n}$ :

$$
\begin{aligned}
& n=1: a_{3}=\frac{\beta^{2}}{3} a_{1}, \text { but } a_{1}=0, \text { and therefore } a_{3}=0 \\
& n=2: a_{4}=\frac{\beta^{2}}{8} a_{2}, \text { where } a_{2} \text { is an arbitrary constant not equal to zero; } \\
& n=3: a_{5}=\frac{\beta^{2}}{15} a_{3}=0, \text { from which we conclude } \\
& \qquad a_{1}=a_{3}=a_{5}=a_{2 n+1}=0 \\
& n=4: a_{6}=\frac{\beta^{2}}{24} a_{4}=\frac{\beta^{4}}{192} a_{2} .
\end{aligned}
$$

Therefore, the solution for the case $\alpha=0$ takes the form

$$
\begin{gathered}
\delta p_{*}(z, 0)=\sum_{n=0}^{\infty} a_{n} z^{n}=a_{0}+a_{1} z+a_{2} z^{2}+a_{3} z^{3}+a_{4} z^{4}+\cdots \\
\delta p_{*}(z, 0)=a_{0}+a_{2} z^{2}+\frac{\beta^{2}}{8} a_{2} z^{4}+\frac{\beta^{2}}{192} a_{2} z^{6}+\cdots=a_{0}+a_{2}\left(z^{2}+\frac{\beta^{2}}{8} z^{4}+\frac{\beta^{2}}{192} z^{6}+\cdots\right) .
\end{gathered}
$$

Let

$$
\begin{gathered}
p(z)=-1=\sum_{n=0}^{\infty} p_{n} Z^{n}, \text { which implies } p_{0}=-1, p_{i}=0 \forall i \neq 0, \\
q(z)=-\beta^{2} Z^{2}=\sum_{n=0}^{\infty} q_{n} Z^{n}, \text { which implies } q_{2}=-\beta^{2}, q_{i}=0 \forall i \neq z .
\end{gathered}
$$

Checking the Bender/Orszag equality,

$$
\begin{gathered}
0 a_{N}=-\sum_{k=0}^{N-1}\left[(\alpha+k) p_{N-K}+q_{N-K}\right] a_{k}, \\
\alpha=0, \quad N=\alpha_{1}-\alpha_{2}=2-0=2, \\
0 a_{2}=-\sum_{k=0}^{1}\left[k p_{2-k}+q_{2-k}\right] a_{k}=-a_{0}-\left[p_{1}+q_{1}\right] a_{1}=\beta^{2} a_{0} \neq 0 .
\end{gathered}
$$


This is case II(b) (I). Therefore, there exists a second solution, not of Frobenius type,

$$
\begin{gathered}
y(z)=\sum_{n=0}^{\infty} c_{n} z^{n}-\left.\frac{\partial}{\partial \alpha} y(z, \alpha)\right|_{\alpha=2}, \\
y(z, \alpha)=\sum_{n=0}^{\infty} a_{n}(\alpha) z^{n+\alpha},
\end{gathered}
$$

where

$$
\begin{gathered}
a_{n}(\alpha)=\frac{-\sum_{k=0}^{n-1}\left[(\alpha+k) p_{n-k}+q_{n-k}\right] a_{k}}{(\alpha+n)^{2}+\left(p_{0}-1\right)(\alpha+n)+q_{0}}, \quad n=1,2,3, \ldots, \\
\quad p(\alpha)=\alpha^{2}-2 \alpha \text { (indicial polynomial), } \\
c_{n}=\frac{-\sum_{k=0}^{n-1}\left(k p_{n-k}+q_{n-k}\right) c_{k}}{p(n)}, \quad n \neq 0, N,
\end{gathered}
$$

where $c_{0}, c_{N}$ are arbitrary and

$$
a_{0}=\frac{1}{p^{\prime}\left(\alpha_{1}\right)} \sum_{k=0}^{N-1}\left[k p_{N-k}+q_{N-k} c_{k}\right], \quad a_{0} \neq 0 .
$$

Upon substitution of the above values in (B8a), we get, for the second solution not of Frobenius type,

$$
\delta p_{*}(z)=a_{0}+a_{2}\left(z^{2}+\frac{\beta^{2}}{8} z^{4}+\cdots\right)+a_{0} \ln Z\left[z^{2}+\frac{\beta^{2}}{32} z^{4}-\frac{\beta^{4} z^{6}}{1152}+\cdots\right] \quad\left(z=u_{A}-1\right) .
$$

The solution to (B1) about the singularity $u_{A}=1$ is a linear combination of (B9) and (B10) with $z$ replaced by $u_{A}-1$ or $\delta p_{*}\left(u_{A}\right)=c_{1} \delta p_{*}\left(u_{A}-1,0\right)+c_{2} \delta p_{*}\left(u_{A}-1,2\right)$ where $c_{1}, c_{2}$ are arbitrary constants and

$$
\begin{gathered}
\delta p_{*}\left(u_{A}-1,0\right)=a_{0}+a_{2}\left(\left(u_{A}-1\right)^{2}+\frac{\beta^{2}}{8}\left(u_{A}-1\right)^{4}+\frac{\beta^{2}}{192}\left(u_{A}-1\right)^{6}+\cdots\right), \\
\delta p_{*}\left(u_{A}-1,2\right)=a_{2} \cdot\left(\left(u_{A}-1\right)^{2}+\frac{\beta^{2}}{8}\left(u_{A}-1\right)^{4}+\frac{\beta^{2}}{192}\left(u_{A}-1\right)^{6}\right. \\
\left.+\frac{\beta^{6}\left(u_{A}-1\right)^{8}}{9216}+\frac{\beta^{8}\left(u_{A}-1\right)^{8}}{737280}+\cdots\right)+a_{0} \ln \left(u_{A}-1\right)\left[\left(u_{A}-1\right)^{2}+\frac{\beta^{2}}{32}\left(u_{A}-1\right)^{4}+\cdots\right] .
\end{gathered}
$$

Next, we look at the solutions of (B1) about the singularity corresponding to the vanishing of $\left(1+q^{2}\right) u_{A}^{2}-1=0$, or $u_{A}=1 / \sqrt{1+q^{2}}$. Our approach will be the same as above. Dividing both sides of (B1) by $M_{A}^{2}$ and setting $k^{2} / M_{A}^{2}=\beta^{2}$, we have

$$
\frac{d^{2}\left(\delta p_{*}\right)}{d u_{A}^{2}}-\frac{2 u_{A}}{u_{A}^{2}-1} \frac{d\left(\delta p_{*}\right)}{d u_{A}}=\beta^{2}\left[\frac{\left(1+q^{2}\right) u_{A}^{2}-1-u^{2} u_{A}^{2}}{\left(1+q^{2}\right) u_{A}^{2}-1}\right] \delta p_{*} .
$$


We simplify (B12) by setting $u_{A}=1 / \sqrt{1+q^{2}}$ everywhere but in the denominator of the right-hand side. Using

$$
\begin{gathered}
\frac{2 u_{A}}{u_{A}^{2}-1}=\frac{2\left(\frac{1}{\sqrt{1+q^{2}}}\right)}{\frac{1}{1+q^{2}}-1}=\frac{-2 \sqrt{1+q^{2}}}{q^{2}}, \\
\left(1+q^{2}\right) u_{A}^{2}-1=\left(\sqrt{1+q^{2}} u_{A}-1\right)\left(\sqrt{1+q^{2}} u_{A}+1\right) .
\end{gathered}
$$

Setting $\sqrt{1+q^{2}} u_{A}=1$, we get $\left(1+q^{2}\right) u_{A}^{2}-1=2\left(\sqrt{1+q^{2}} u_{A}-1\right)$, or

$$
\left(1+q^{2}\right) u_{A}^{2}-1=2 \sqrt{1+q^{2}}\left(u_{A}-\frac{1}{\sqrt{1+q^{2}}}\right) \text {. }
$$

We further simplify the numerator of the right-hand side of (B12) to

$$
\left(1+q^{2}\right) u_{A}^{2}-1-u^{2} u_{A}^{2}=1-1-u^{2} u_{A}^{2} .
$$

Note that $u_{A}=\frac{k}{k_{z}} \frac{u}{q}$. Solving for $u$ we get $u=\frac{k_{z}}{k} q u_{A}$ and, substituting in the above, the numerator of the right-hand side simplifies further to

$$
\left(1+q^{2}\right) u_{A}^{2}-1-u^{2} u_{A}^{2}=-\frac{k_{z}^{2}}{k^{2}} q^{2} u_{A}^{4}=\frac{-k_{z}^{2}}{k^{2}} q^{2} \frac{1}{\left(1+q^{2}\right)^{2}}=\frac{-\left(k_{z}^{2} / k^{2}\right) q^{2}}{\left(1+q^{2}\right)^{2}} .
$$

Substituting (B14) and (B13c) into the right-hand side of (B12), this further simplifies to

$$
\frac{\left(-k^{2} / k_{z}^{2}\right) q^{2}}{\left(1+q^{2}\right)^{2}} /\left(2\left(1+q^{2}\right)^{1 / 2}\left(u_{A}-\frac{1}{\sqrt{1+q^{2}}}\right)\right)=\frac{-k^{2} q^{2}}{2 k_{z}^{2}\left(1+q^{2}\right)^{5 / 2}} \times \frac{1}{u_{A}-\frac{1}{\sqrt{1+q^{2}}}} .
$$

Setting $-\beta^{2} k^{2} q^{2} /\left(2 k_{z}^{2}\left(1+q^{2}\right)^{5 / 2}\right)=\sigma$ and $-2 \sqrt{1+q^{2}} / q^{2}=\tau$ and substituting in (B12) we get

$$
\begin{gathered}
\frac{-2 u_{A}}{u_{A}^{2}-1}=\frac{2 \sqrt{1+q^{2}}}{q^{2}}=-\tau \\
\beta^{2}\left[\frac{\left(1+q^{2}\right) u_{A}^{2}-1-u^{2} u_{A}^{2}}{\left(1+q^{2}\right) u_{A}^{2}-1}\right]=\frac{-\beta^{2} k^{2} q^{2}}{2 k_{z}^{2}\left(1+q^{2}\right)^{5 / 2}} \frac{1}{u_{A}-1 / \sqrt{1+q^{2}}}=\frac{\sigma}{u_{A}-1 / \sqrt{1+q^{2}}} .
\end{gathered}
$$

Substituting (B15) and (B16) into (B12) yields

$$
\frac{d^{2}\left(\delta p_{*}\right)}{d u_{A}^{2}}-\tau \frac{d\left(\delta p_{*}\right)}{d u_{A}}-\frac{\sigma}{u_{A}-1 / \sqrt{1+q^{2}}} \delta p_{*}=0
$$

Setting $z=u_{A}-1 / \sqrt{1+q^{2}}$, Eq. (B17) simplifies to

$$
\frac{d^{2}\left(\delta p_{*}\right)}{d z^{2}}-\tau \frac{d\left(\delta p_{*}\right)}{d z}-\frac{\sigma}{z} \delta p_{*}=0
$$


Equation (B18) has a regular singular point about $z=0$. We assume a Frobenius type of solution. Set $\delta p_{*}=\sum_{n=0}^{\infty} a_{n} z^{n+\alpha}$, where $\alpha$ is the indicial exponent to be determined. Substituting the series expression for $\delta p_{*}$ in (B18) we get

$$
\begin{gathered}
\frac{d\left(\delta p_{*}\right)}{d z}=\sum_{n=0}^{\infty} a_{n}(n+\alpha) z^{n+\alpha-1}, \quad \frac{d^{2}\left(\delta p_{*}\right)}{d z^{2}}=\sum_{n=0}^{\infty} a_{n}(n+\alpha)(n+\alpha-1) z^{n+\alpha-2}, \\
\sum_{n=0}^{\infty} a_{n}(n+\alpha)(n+\alpha-1) z^{n+\alpha-2}-\tau \sum_{n=0}^{\infty} a_{n}(n+\alpha) z^{n+\alpha-1} \\
-\sigma \sum_{n=0}^{\infty} a_{n} z^{n+\alpha-1}=0
\end{gathered}
$$

The first term can be written in the form

$$
\begin{aligned}
\sum_{n=0}^{\infty} a_{n}(n+\alpha)(n+\alpha-1) z^{n+\alpha-2}=a_{0} \alpha(\alpha-1) z^{\alpha-2} & \\
& +\sum_{n=0}^{\infty} a_{n+1}(n+\alpha)(n+\alpha+1) z^{n+\alpha-1} .
\end{aligned}
$$

Substituting the above expression in (B19) yields

$$
\alpha_{0} \alpha(\alpha-1) z^{\alpha-2}+\sum_{n=0}^{\infty}\left[a_{n+1}(n+\alpha)(n+\alpha+1)-\tau(n+\alpha) a_{n}-\sigma a_{n}\right] z^{n+\alpha-1}=0 .
$$

We set the coefficient powers of $z^{\alpha-2}$ and $z^{n+\alpha-1}$ equal to zero, $a_{0} \alpha(\alpha-1)=0$. Our assumption is $a_{0} \neq 0$ and, therefore, $\alpha(\alpha-1)=0$, which implies $\alpha_{1}=1, \alpha_{2}=0$, and

$$
\alpha_{n+1}(n+\alpha)(n+\alpha+1)-[\tau(n+\alpha)+\sigma] a_{n}=0 .
$$

Solving for $a_{n+1}$,

$$
a_{n+1}=\frac{(\tau(n+\alpha)+\sigma) a_{n}}{(n+\alpha)(n+\alpha+1)} .
$$

Two cases are to be considered: $\alpha_{1}=1$ and $\alpha_{2}=0$.

For $\alpha=1$, the recursion relation

$$
a_{n+1}=\frac{(\tau(n+\alpha)+\sigma)}{(n+\alpha)(n+\alpha+1)} a_{n}
$$

can be written in the form

$$
a_{n+1}=\frac{(\tau(n+1)+\sigma)}{(n+1)(n+2)} a_{n}
$$

where $a_{0} \neq 0$ :

$$
\begin{aligned}
& n=0: a_{1}=\frac{(\tau+\sigma)}{2} a_{0} \\
& n=1: a_{2}=\frac{(2 \tau+\sigma)}{6} a_{1}=\frac{(2 \tau+\sigma)(\tau+\sigma)}{12} a_{0} \\
& n=2: a_{3}=\frac{(3 \tau+\sigma)}{12} a_{2}=\frac{(3 \tau+\sigma)(2 \tau+\sigma)(\tau+\sigma)}{144} a_{0}
\end{aligned}
$$


Continuing in this fashion, all the $a_{i}$ 's can be written as a multiple of $a_{0}$. Therefore, the Frobenius solution corresponding to the case $\alpha=1$ has the form

$$
\delta p_{*}=\sum_{n=0}^{\infty} a_{n} z^{n+1}=a_{0} z^{1}+a_{1} z^{2}+a_{2} z^{3}+a_{3} z^{4}+\cdots
$$

Substituting the values of $a_{0}, a_{1}, a_{2}, a_{3}$ in the above expression, we get

$$
\begin{gathered}
\delta p_{*}=a_{0} z+\frac{\tau+\sigma}{2} a_{0} z^{2}+\frac{(2 \tau+\sigma)(\tau+\sigma)}{12} a_{0} z^{3}+\frac{(3 \tau+\sigma)(2 \tau+\sigma)(\tau+\sigma)}{144} a_{0} z^{4}+\cdots \\
\delta p_{*}=\left(z+\frac{\tau+\sigma}{2} z^{2}+\frac{(\tau+\sigma)(2 \tau+\sigma)}{12} z^{3}+\frac{(\tau+\sigma)(2 \tau+\sigma)(3 \tau+\sigma)}{144} z^{4}+\cdots\right) a_{0}
\end{gathered}
$$

We rewrite (B18) in the following form:

$$
\begin{gathered}
\frac{d^{2}\left(\delta p_{*}\right)}{d z^{2}}-\frac{\tau z}{z} \frac{d}{d z}\left(\delta p_{*}\right)-\frac{\sigma z}{z^{2}} \delta p_{*}=0 \\
p(z)=-\tau z, \quad q(z)=-\sigma z .
\end{gathered}
$$

Expressing $p(z)$ and $q(z)$ in power series:

$$
p(z)=\sum_{n=0}^{\infty} p_{n} z^{n}=p_{0}+p_{1} z+p_{2} z^{2}+\cdots=-\tau z
$$

which implies

$$
p_{0}=p_{2}=p_{3}=\cdots=0, \quad p_{1}=-\tau .
$$

Expanding $q(z)$ in a Taylor series:

$$
q(z)=\sum_{n=0}^{\infty} q_{n} z^{n}=q_{0}+q_{1} z+q_{2} z^{2}+q_{3} z^{3}+\cdots=-\sigma z
$$

yields

$$
q_{0}=q_{2}=q_{3}=\cdots=0, \quad q_{1}=-\sigma .
$$

We check the recursion relation (Bender and Orszag, 1978):

$$
0 a_{N}=-\sum_{k=0}^{N-1}\left[(\alpha+k) p_{N-k}+q_{N-k}\right] a_{k}, \quad N=\alpha_{1}-\alpha_{2}=1, \quad \alpha=\alpha_{2}=0
$$

or

$$
0 a_{N}=-\sum_{k=0}^{0}\left[k p_{1-k}+q_{1-k}\right] a_{k}=-q_{1} a_{0}
$$

From our assumption for the existence of a Frobenius solution, $a_{0} \neq 0, q_{1}=-\sigma \neq 0$. Therefore, the right side is nonzero. This is the case II (b) in Bender and Orszag (1978), 
Chapter 3. To find the second solution to (B18) we follow the discussion on p. 75 of Bender and Orszag. The second solution has the form

$$
\delta p_{*}(z)=\sum_{n=0}^{\infty} c_{n} z^{n}-\left.\frac{\partial}{\partial \alpha} \delta p_{*}(z, \alpha)\right|_{\alpha=\alpha_{1}}
$$

where $c_{0}$ and $c_{1}$ are arbitrary, and

$$
\begin{aligned}
c_{n}= & \frac{-\sum_{k=0}^{n-1}\left[\left(\alpha_{2}+k\right) p_{n-k}+q_{n-k}\right] c_{k}}{p\left(\alpha_{2}+n\right)}, \quad \alpha_{2}=0, \quad p(\alpha)=\alpha^{2}-\alpha \\
c_{n} & =\frac{-\sum_{k=0}^{n-1}\left(k p_{n-k}+q_{n-k}\right) c_{k}}{\left(n^{2}-n\right)},
\end{aligned}
$$

$n=2: c_{2}=\frac{-\sum_{k=0}^{1}\left(k p_{2-k}+q_{2-k}\right) c_{k}}{2}=\frac{-q_{2} c_{0}-\left(p_{1}+q_{1}\right) c_{1}}{2}=\frac{(\tau+\sigma) c_{1}}{2}$,

$n=3: c_{3}=\frac{-\sum_{k=0}^{2}\left(k p_{3-k}+q_{3-k}\right) c_{k}}{6}=\frac{-\left(2 p_{1}+q_{1}\right) c_{2}}{6}=\frac{(2 \tau+\sigma) c_{2}}{6}=\frac{(\tau+\sigma)(2 \tau+\sigma) c_{1}}{12}$.

To calculate the second term of (B23), we write the expression for $p$ and then take its derivative with respect to $\alpha, \delta p_{*}(z, \alpha)=\sum_{n=0}^{\infty} a_{n}(\alpha) z^{n+\alpha}$, where

$$
a_{n}(\alpha)=\frac{-\sum_{k=0}^{n-1}\left[(\alpha+k) p_{n-k}+q_{n-k}\right] a_{k}}{\left[(\alpha+n)^{2}-(\alpha+n)\right]}
$$

and

$$
a_{0}=\frac{1}{p^{\prime}\left(\alpha_{1}\right)} \sum_{k=0}^{N-1}\left[\left(\alpha_{2}+k\right) p_{N-k}+q_{N-k}\right] c_{k} .
$$

Since $p(\alpha)=\alpha^{2}-\alpha$, taking its derivative yields $p^{\prime}(\alpha)=2 \alpha-1$. Setting $\alpha=\alpha_{1}=1$, we get $p^{\prime}\left(\alpha_{1}\right)=1, N=\alpha_{1}-\alpha_{2}=1$. Plugging these values in (B25),

$$
a_{0}=\sum_{k=0}^{\infty}\left[k p_{1-k}+q_{1-k}\right] c_{k}=q_{1} c_{0}
$$

But, $q_{1}=-\sigma$; hence, $a_{0}=-\sigma c_{0}$. Taking the derivative of $\delta p_{*}(z, \alpha)=\sum_{n=0}^{\infty} a_{n}(\alpha) z^{n+\alpha}$ with respect to $\alpha$, we get

$$
\left.\frac{\partial}{\partial \alpha} \delta p_{*}(z, \alpha)\right|_{\alpha=\alpha_{1}}=\ln z \sum_{n=0}^{\infty} a_{n}\left(\alpha_{1}\right) z^{n+\alpha_{1}}+\left.\sum_{n=0}^{\infty} \frac{\partial}{\partial \alpha} a_{n}(\alpha)\right|_{\alpha=\alpha_{1} \times z^{\alpha_{1}+n}}
$$


We further try to simplify expression (B26). Using (B24),

$$
a_{n}\left(\alpha_{1}\right)=a_{n}(1)=\frac{-\sum_{k=0}^{n-1}\left[(1+k) p_{n-k}+q_{n-k}\right] a_{k}}{(1+n)^{2}-(n+1)}=\frac{-\sum_{k=0}^{n-1}\left[(1+k) p_{n-k}+q_{n-k}\right] a_{k}}{n(n+1)} .
$$

We calculate some coefficients of $a_{n}(1)$ :

$$
n=1: a_{1}(1)=\frac{-\sum_{k=0}^{0}\left[(1+k) p_{1-k}+q_{1-k}\right] a_{k}}{2}=\frac{-\left(p_{1}+q_{1}\right) a_{0}}{2}, \quad p_{1}=-\tau, q_{1}=-\sigma .
$$

Therefore, $a_{1}(1)=(\tau+\sigma) a_{0} / 2$ but $a_{0}=-\sigma c_{0}$, and so $a_{1}(1)=-(\tau+\sigma) \sigma c_{0} / 2$. Let $n=2$ in (B24). Then

$$
a_{2}(1)=\frac{-\sum_{k=0}^{1}\left[(1+k) p_{2-k}+q_{2-k}\right] a_{k}}{6},
$$

which upon expansion gives

$$
\begin{gathered}
a_{2}(1)=\frac{-\left[0_{2}+0_{2}\right] a_{0}-\left[2 p_{1}+q_{1}\right] a_{1}}{6}=\frac{-\left[2 p_{1}+q_{1}\right] a_{1}}{6}, \\
p_{1}=-\tau, \quad q_{1}=-\sigma, \quad a_{1}=\frac{\tau+\sigma}{2} a_{0}, \quad a_{2}=\frac{(2 \tau+\sigma)(\tau+\sigma) a_{0}}{12} .
\end{gathered}
$$

For $n=3$, we have

$$
\begin{gathered}
a_{3}=\frac{-\sum_{k=0}^{2}\left[(1+k) p_{3-k}+q_{3-k}\right] a_{k}}{12}=\frac{-\left[\left(0_{3}+0_{3}\right) a_{0}+\left(20_{2}+0_{2}\right) a_{1}+\left(3 p_{1}+q_{1}\right) a_{2}\right]}{12}, \\
a_{3} \frac{-\left(3 p_{1}+q_{1}\right) a_{2}}{12}=\frac{(3 \tau+\sigma) a_{2}}{12}=\frac{(3 \tau+\sigma)(2 \tau+\sigma)(\tau+\sigma) a_{0}}{144} .
\end{gathered}
$$

It can be concluded from the above calculations that $a_{n}$ can be written in the form

$$
a_{n}=\frac{\left[\prod_{i=1}^{i=n}(\sigma+i \tau)\right] a_{0}}{(2 n)^{n-1}(n+1)}
$$

Therefore, the first term of (B26) has the following expansion:

$$
\ln z \sum_{n=0}^{\infty} a_{n}\left(\alpha_{1}\right) z^{n+1}=\ln z\left[a_{0} z+a_{1}(1) z^{2}+a_{2}(1) z^{3}+a_{3}(1) z^{4}+\cdots\right] .
$$

Substituting the above values of $a_{1}(1), a_{2}(1), a_{3}(1)$, we get

$$
\ln z \sum_{n=0}^{\infty} a_{n}(1) z^{n+1}=\ln z\left[z-\frac{(\tau+\sigma)}{2} z^{2}+\frac{\prod_{i=1}^{2}(\sigma+i \tau)}{12} z^{3}+\frac{\prod_{i=1}^{3}(\sigma+i \tau)}{144} z^{4}+\cdots\right] a_{0}
$$


We try to simplify the second term of (B26). We need to calculate $\left.\frac{\partial}{\partial \alpha} a_{n}(\alpha)\right|_{\alpha=\alpha_{1}}$ for different $n$. With $a_{0} \neq 0$,

$$
a_{n}(\alpha)=\frac{-\sum_{k=0}^{n-1}\left[(\alpha+k) p_{n-k}+q_{n-k}\right] a_{k}}{(\alpha+n)^{2}-(\alpha+n)}
$$

Since $q_{i}=0 \forall i \neq 1, q_{1}=-\sigma, p_{i}=0 \forall i \neq 1, p_{1}=-\tau$, the expression for $a_{n}(\alpha)$ simplifies to

$$
a_{n}(\alpha)=\frac{-\left((\alpha+n-1) p_{1}+q_{1}\right) a_{n-1}}{(\alpha+n)^{2}-(\alpha+n)}=\frac{((\alpha+n-1) \tau+\sigma) a_{n-1}}{(\alpha+n)^{2}-(\alpha+n)}, \quad n=1,2,3,
$$

and $a_{0} \neq 0$ is an arbitrary constant. Also,

$$
\begin{gathered}
n=1: a_{1}(\alpha)=\frac{\alpha \tau+\sigma}{\alpha(\alpha+1)} a_{0},\left.\quad \frac{\partial a_{1}(\alpha)}{\partial \alpha}\right|_{\alpha=1}=-\frac{(\tau+3 \sigma)}{4} a_{0}, \\
n=2: a_{2}(\alpha)=\frac{((\alpha+1) \tau+\sigma) a_{1}}{(\alpha+2)(\alpha+1)}, \text { and replacing } a_{1}=\frac{\alpha \tau+\sigma}{\alpha(\alpha+1)} a_{0} \text { we get } \\
a_{2}(\alpha)=\frac{\left(\alpha^{2}+\alpha\right) \tau^{2}+(2 \alpha+1) \tau \sigma+\sigma^{2}}{\alpha(\alpha+1)^{2}(\alpha+2)} a_{0},\left.\quad \frac{\partial a_{2}(\alpha)}{\partial \alpha}\right|_{\alpha=1}=\frac{-\left(5 \tau^{2}+15 \tau \sigma+7 \sigma^{2}\right)}{36} a_{0} .
\end{gathered}
$$

Using the same method, we calculate other terms, and we will have more complicated expressions in terms of $\tau$ and $\sigma$. The second term of (B26), upon substitution of the above values of $\left.\frac{\partial}{\partial \alpha} a_{1}(\alpha)\right|_{\alpha=1},\left.\frac{\partial}{\partial \alpha} a_{2}(\alpha)\right|_{\alpha=1}$, has the following form:

$$
\left.\sum_{n=0}^{\infty} \frac{\partial}{\partial \alpha} a_{n}(\alpha)\right|_{\alpha=1} z^{1+n}=\left.\frac{\partial}{\partial \alpha} a_{0}(\alpha)\right|_{\alpha=1} z+\left.\frac{\partial}{\partial \alpha} a_{1}(\alpha)\right|_{\alpha=1} z^{2}+\left.\frac{\partial}{\partial \alpha} a_{2}(\alpha)\right|_{\alpha=1} z^{3}+\cdots
$$

where $a_{0}$ is an arbitrary constant or $\frac{\partial}{\partial \alpha} a_{0}(\alpha)=0$ and

$$
\left.\sum_{n=0}^{\infty} \frac{\partial}{\partial \alpha} a_{n}(\alpha)\right|_{\alpha=1} z^{1+n}=\left(-\frac{(\tau+3 \sigma)}{4} z^{2}-\frac{\left(5 \tau^{2}+15 \tau \sigma+7 \sigma^{2}\right)}{36} z^{3}+\cdots\right) a_{0}
$$

Substituting (B27) and (B28) into (B26) we get

$$
\begin{aligned}
& \left.\frac{\partial}{\partial \alpha} \delta p_{*}(z, \alpha)\right|_{\alpha=1} \\
& =\ln z\left[z-\frac{(\tau+\sigma)}{2} z^{2}+\frac{(2 \tau+\sigma)(\tau+\sigma)}{12} z^{3}+\frac{(\tau+\sigma)(\sigma+2 \tau)(\sigma+3 \tau)}{144} z^{4}+\cdots\right] a_{0} \\
& +\left(-\frac{(\tau+3 \sigma)}{4} z^{2}-\left(\frac{5 \tau^{2}+15 \tau \sigma+7 \sigma^{2}}{36}\right) z^{3}+\cdots\right) a_{0} .
\end{aligned}
$$


We expand the first term of (B23) as follows:

$$
\begin{gathered}
\sum_{n=0}^{\infty} c_{n} z^{n}=c_{0}+c_{1} z+c_{2} z^{2}+c_{3} z^{3}+\cdots, \\
\sum_{\substack{n=0 \\
\infty}}^{\infty} c_{n} z^{n}=c_{0}+c_{1} z+\frac{(\tau+\sigma)}{2} c_{1} z^{2}+\frac{(\tau+\sigma)(2 \tau+\sigma)}{12} c_{1} z^{3}+\cdots \\
\sum_{n=0}^{\infty} c_{n} z^{n}=c_{0}+c_{1}\left(z+\frac{(\tau+\sigma)}{2} z^{2}+\frac{(\tau+\sigma)(2 \tau+\sigma)}{12} z^{3}+\cdots\right) .
\end{gathered}
$$

If $c_{0}$ and $c_{1}$ are arbitrary nonzero constants, substituting (B29) and (B30) into (B23), we get the local series expansion of (B18) about the singularity $u_{A}=1 / \sqrt{1+q^{2}}(z=0)$ :

$$
\begin{aligned}
\delta p_{*}(z) & =c_{0}+c_{1}\left(z+\frac{(\tau+\sigma)}{2} z^{2}+\frac{(\tau+\sigma)(2 \tau+\sigma)}{12} z^{3}+\cdots\right) \\
-\ln z\left[z-\left(\frac{\tau+\sigma}{2}\right) z^{2}\right. & \left.+\frac{(2 \tau+\sigma)(\tau+\sigma)}{12} z^{3}+\frac{(\tau+\sigma)(\sigma+2 \tau)(\sigma+3 \tau)}{144} z^{4}+\cdots\right] a_{0} \\
& -\left(-\frac{(\tau+3 \sigma)}{4} z^{2}-\frac{\left(5 \tau^{2}+15 \tau \sigma+\tau \sigma^{2}\right)}{36} z^{3}+\cdots\right) a_{0},
\end{aligned}
$$

where $a_{0}=-\sigma c_{0}$, and $z=u_{A}-1 / \sqrt{1+q^{2}}$.

\section{REFERENCES}

[1] H. Aref and E. D. Siggia, Evolution and breakdown of a vortex street in two dimensions, J. Fluid Mech. 109, 435-463 (1981)

[2] H. Aref and G. Tryggvason, Dynamics of passive and active interfaces, Physica 2D. 59-70 (1984)

[3] M. C. Begelman, R. D. Blandford, and M. J. Rees, Theory of extragalactic radio sources, Rev. Modern Phys. 56, 255 -351 (1984)

[4] C. M. Bender and S. Orszag, Advanced Mathematical Methods for Scientists and Engineers, McGraw-Hill, New York, 1978

[5] R. D. Blandford and J. E. Pringle, Kelvin-Helmholtz instability of relativistic beams, Monthly Notices Roy. Astron. Soc. 176, 443-454 (1976)

[6] W. Blumen, Shear layer instability of an inviscid compressible fluid, J. Fluid Mech. 40, 769-781 (1970)

[7] W. Blumen, P. G. Drazin, and D. F. Billings, Shear layer instability of an inviscid compressible fluid, Part 2, J. Fluid Mech. 71, 305316 (1975)

[8] J. C. Brandt and D. A. Mendis, The solar wind, in Solar System Plasma Physics, edited by C. F. Kennel, L. Lanzerotti, and E. N. Parker, Amsterdam, North-Holland, 1979

[9] H. S. Bridge, J. W. Belcher, A. J. Lazarus, J. D. Sullivan, F. Bagenal, R. L. McNutt, Jr., K. W. Ogilvie, J. D. Scudder, and E. C. Sittler, Plasma observations near Jupiter: Initial results, Science 206, 972-976 (1979)

[10] G. L. Brown and A. Roshko, On density effects and large structure in turbulent mixing layers, J. Fluid Mech. 64, 775-816 (1974)

[11] K. M. Case, Stability of inviscid plane Couette flow, Phys. Fluids 3, 143-148; and Stability of an idealized atmosphere I. Discussion of results, Phys. Fluids 3, 149-157 (1960)

[12] S. Chandrasekhar, Hydrodynamic and Hydromagnetic Stability, Dover (originally published 1961, Oxford, Clarendon), New York, 1981

[13] H. Dobrowolny and N. D'Angelo, Wave motion in type I comet tails, in Cosmic Plasma Physics (K. Schindler, ed.), Plenum, New York, 1972 
[14] A. I. Ershkovich, A. A. Nusmov, and A. A. Chernikov, Oscillations of type I comet tails, Planetary and Space Science 20, 1235-1243 (1972)

[15] J. A. Fejer, Hydromagnetic stability at a fluid velocity discontinuity between compressible fluids, Phys. Fluids 7, 499-503 (1964)

[16] R. A. Gerwin, Stability of the interface between two fluids in relative motion, Rev. Modern Phys. 40, 652-658 (1968)

[17] E. L. Ince, Ordinary Differential Equations, Dover, New York, 1956

[18] J. R. Jokipii and L. Davis, Long wavelength turbulence and the heating of the solar wind, Astrophysical J. 156, 1101-1106 (1969)

[19] K. J. Kaup, Coherent structures in the planar magnetron, Phys. Fluids B2, 2253-2258 (1990)

[20] N. A. Krall and A. W. Trivelpiece, Principles of Plasma Physics, McGraw-Hill, New York, 1973

[21] L. D. Landau, The instability of moving superposed fluids, Akad. Nauk. S.S.S.R., Comptes Rendus (Doklady) 44, 139-144 (1944)

[22] T. N. Larosa and R. L. Moore, A mechanism for bulk energization in the impulsive phase of solar flares, Astrophysical J. 418, 912-918 (1993)

[23] I. Lerche, Validity of the hydromagnetic approach in discussing instability of the magnetospheric boundary, J. Geophysical Res. 71, 2365-2371 (1966)

[24] A. Michalke, On the inviscid instability of the hyperbolic-tangent velocity profile, J. Fluid Mech. 19, 543-556 (1964)

[25] J. W. Miles, On wind over water, J. Acoustical Soc. America 29, 226-230 (1957)

[26] J. W. Miles, On the disturbed motion of a plane vortex sheet, J. Fluid Mech. 4, 538-552 (1958)

[27] A. Miura and P. L. Pritchett, Nonlocal stability analysis of the MHD Kelvin-Helmholtz instability in a compressible plasma, J. Geophysical Res. 87, 7431-7444 (1982)

[28] A. Miura, Anomalous transport by magnetohydrodynamic Kelvin-Helmholtz instabilities in the solar wind-magnetosphere interaction, J. Geophysical Res. 89, 801-818 (1984)

[29] M. Nepveu, Cylindrical jets, Astronomy and Astrophysics 84, 14-21 (1980)

[30] N. F. Ness, M. H. Acuna, R. P. Lepping, J. E. P. Connerney, K. W. Behannon, and L. F. Burlaga, Magnetic field studies by Voyager I, Science 212, 211-217 (1981)

[31] M. L. Norman, L. Smarr, K. H. A. Winkler, and M. D. Smith, Instabilities of cylindrical jets, Astronomy and Astrophysics 113, 285-351 (1982)

[32] E. N. Parker, Interplanetary Dynamical Processes, Interscience, New York, 1963

[33] P. L. Pritchett and F. V. Coroniti, The collisionless macroscopic Kelvin-Helmholtz instability 1. Transverse electrostatic mode, J. Geophysical Res. 89, 168-178 (1984)

[34] Z. Y. Pu and M. G. Kivelson, Kelvin-Helmholtz instability at the magnetopause: Solution for compressible plasmas, J. Geophysical Res. 88, 841-852; and, Energy flux into the magnetosphere, J. Geophysical Res. 88, 853-861 (1983)

[35] T. P. Ray, The effects of a simple shear layer on the growth of Kelvin-Helmholtz instabilities, Monthly Notices Roy. Astron. Soc. 198, 617-625 (1982)

[36] T. P. Ray and A. I. Ershkovich, Kelvin-Helmholtz instabilities of magnetized shear layers, Monthly Notices Roy. Astron. Soc. 204, 821--826 (1983)

[37] S. Roy Choudhury and R. V. E. Lovelace, On the Kelvin-Helmholtz instabilities of supersonic shear layers, Astrophysical J. 283, 331-342 (1984)

[38] S. Roy Choudhury and R. V. E. Lovelace, On the Kelvin-Helmholtz instabilities of high-velocity magnetized shear layers, Astrophysical J. 302, 188-199 (1986)

[39] S. Roy Choudhury, Kelvin-Helmholtz instabilities of supersonic, magnetized shear layers, J. Plasma Phys. 35, 375-392 (1986)

[40] S. Roy Choudhury and V. L. Patel, Kelvin-Helmholtz instabilities of high-velocity, magnetized anisotropic shear layers, Phys. Fluids 28, 3292-3301 (1985)

[41] S. Roy Choudhury, Global asymptotic analysis of the Kelvin-Helmholtz instability of supersonic shear layers, Canadian J. Phys. 68, 334-342 (1990)

[42] A. K. Sen, Stability of the magnetospheric boundary, Planetary and Space Science 13, 131-141 (1965)

[43] A. K. Sen, Effect of compressibility on Kelvin-Helmholtz instability in a plasma, Phys. Fluids $\mathbf{7}$, 1293-1298 (1964)

[44] D. J. Southwood, The hydromagnetic stability of the magnetospheric boundary, Planetary and Space Science 16, 587-605 (1968) 
[45] D. J. Southwood, Some features of field-line resonances in the magnetosphere, Planetary and Space Science 22, 483-491 (1974)

[46] P. A. Sturrock and R. E. Hartle, Two-fluid model of the solar wind, Physical Review Lett. 16, 628-631 (1966)

[47] T. Tajima and J. N. Leboeuf, Kelvin-Helmholtz instability in supersonic and super-Alfvenic fluids, Phys. Fluids 23, 884-888 (1980)

[48] S. P. Talwar, Kelvin-Helmholtz instability in an anisotropic plasma, Phys. Fluids 8, 1295-1299 (1965)

[49] B. D. Turland and P. A. G. Scheuer, Instabilities of Kelvin-Helmholtz type for relativistic streaming, Monthly Notices Roy. Astron. Soc. 176, 421-441 (1976)

[50] C. D. Winant and F. K. Browand, Vortex pairing: The mechanism of turbulent mixing-layer growth, J. Fluid Mech. 63, 237-255 (1974) 